\title{
Pyroptosis is driven by non-selective gasdermin-D pore and its morphology is different from MLKL channel-mediated necroptosis
}

Xin Chen ${ }^{1, *}$, Wan-ting $\mathrm{He}^{1, *}$, Lichen $\mathrm{Hu}^{1}$, Jingxian $\mathrm{Li}^{1}$, Yuan Fang ${ }^{1}$, Xin Wang ${ }^{1}$, Xiaozheng Xu ${ }^{1}$, Zhuo Wang ${ }^{1}$, Kai Huang ${ }^{1}$, Jiahuai $\operatorname{Han}^{1}$

${ }^{I}$ State Key Laboratory of Cellular Stress Biology, Innovation Center for Cell Signaling Network, School of Life Sciences, Xiamen University, Xiamen, Fujian 361005, China

Necroptosis and pyroptosis are two forms of programmed cell death with a common feature of plasma membrane rupture. Here we studied the morphology and mechanism of pyroptosis in comparison with necroptosis. Different from necroptosis, pyroptosis undergoes membrane blebbing and produces apoptotic body-like cell protrusions (termed pyroptotic bodies) prior to plasma membrane rupture. The rupture in necroptosis is explosion-like, whereas in pyroptosis it leads to flattening of cells. It is known that the execution of necroptosis is mediated by mixed lineage kinase domain-like (MLKL) oligomers in the plasma membrane, whereas gasdermin-D (GSDMD) mediates pyroptosis after its cleavage by caspase-1 or caspase-11. We show that N-terminal fragment of GSDMD (GSDMD-N) generated by caspase cleavage also forms oligomer and migrates to the plasma membrane to kill cells. Both MLKL and GSDMD-N are lipophilic and the N-terminal sequences of both proteins are important for their oligomerization and plasma membrane translocation. Unlike MLKL which forms channels on the plasma membrane that induces influx of selected ions which osmotically swell the cells to burst, GSDMD-N forms non-selective pores and does not rely on increased osmolarity to disrupt cells. Our study reveals the pore-forming activity of GSDMD and channelforming activity of MLKL determine different ways of plasma membrane rupture in pyroptosis and necroptosis.

Keywords: pyroptosis; necroptosis; GSDMD; MLKL

Cell Research (2016) 26:1007-1020. doi:10.1038/cr.2016.100; published online 30 August 2016

\section{Introduction}

Cell death is a fundamental feature of life and functions in many important biological processes including development, homeostasis and pathogenesis [1]. The forms and mechanisms of cell death are diverse and among them, programmed cell death is proven to be genetically controlled $[2,3]$. Subtypes of cell death have been historically defined by their distinct morphology. Apoptosis, once considered the sole form of programmed cell death, is characterized by cell shrinkage, membrane blebbing and formation of apoptotic bodies $[4,5]$.

\footnotetext{
*These two authors contributed equally to this work.

Correspondence: Jiahuai Han

E-mail: jhan@xmu.edu.cn

Received 10 July 2016; revised 2 August 2016; accepted 3 August 2016; published online 30 August 2016
}

Emerging evidence has now revealed that necrosis can also be genetically controlled and can be classified into several categories [6]. Given the fact that different modes of regulated necrosis share some common morphological features, it is thought that distinguishing them by observing the morphology of dying cells is difficult [7].

Among various forms of programmed necrosis, necroptosis has been intensively investigated and its molecular mechanism is largely known. Necrosomes formed after necroptotic stimulation play a key role in initiating necroptosis. Receptor interacting protein (RIP) 1 and RIP3 are the core components of necrosomes [8-10]. Phosphorylation of MLKL by RIP3 in necrosomes leads to MLKL oligomerization and translocation to the plasma membrane. Once localized in the plasma membrane, MLKL may form permeable ion channels which cause cellular changes that disrupt plasma membrane integrity [11-16]. Pyroptosis is a consequence of caspase- 1 or 
caspase-11 activation in inflammasomes and thus is well known for its function in inflammation [17-19]. Recently GSDMD was identified as an essential factor downstream of caspase- 1 or caspase-11 to mediate pyroptosis and release interleukin (IL)- $1 \beta$ to extracellular space [20-22]. The construction of plasma membrane pores by unknown protein(s) has been described and attributed to the cause of macrophage pyroptosis triggered by lethal toxin and Salmonella infection [23]. Three very recent publications revealed the pore-forming activity of GSDMD N-terminal domain after the release of its C-terminal domain by caspase-1 or caspase-11 cleavage [24-26]. Thus, GSDMD causes pyroptosis by forming pores in the plasma membrane.

To better understand different forms of necrosis, we compared the morphologies and mechanisms of necroptosis and pyroptosis together with the most well studied apoptosis. Although both necroptosis and pyroptosis display plasma membrane disruption which distinguishes them from apoptosis, the morphologies of necroptosis and pyroptosis are also clearly different from each other. Necroptosis and pyroptosis are similar in that the translocation of their executor protein, MLKL and GSDMD, respectively, to the plasma membrane is required for cell death. However, MLKL forms ion selective channels, whereas GSDMD forms pores that lack ion selectivity. These mechanistic differences determine the morphological differences between necroptosis and pyroptosis; and the different ways of plasma membrane rupture suggest that the in vivo functions of necroptosis and pyroptosis are different.

\section{Results}

Pyroptotic and necroptotic cells have distinct morphological features

Necroptosis and pyroptosis have been characterized as programmed cell death with necrotic morphologies such as rupture of plasma membrane [6]. However, detailed morphologic analysis of these two types of cell death is lacking. To compare pyroptosis with necroptosis, we need to use a cell line that can undergo necroptosis and pyroptosis upon different stimulation. RAW-asc cells, a RAW 264.7 cell line ectopically expressing ASC [22], were selected in this study. RAW-asc cells underwent necroptosis upon TNF + smac mimetic + caspase inhibitor z-VAD (TSZ) treatment and pyroptosis upon LPS + nigericin (LPS + Nig) treatment (Figure $1 \mathrm{~B}$ and $1 \mathrm{C}$ ). Apoptosis was also induced in this cell line as we observed time-dependent increase of annexin V-positive staining after TNF + smac mimetic (TS) treatment without propidium iodide (PI) uptake (Figure 1A). The mor- phologies of the cell death were analyzed in real-time by light microscopy or at high resolution by electron microscopy (EM). As expected, TS-treated cells showed classic apoptotic bodies (Figure 1D and 1G, TS). Necroptosis began with a rounding up of the cell body, which was accompanied by a partial detachment of the cell from culture slide, followed by the swelling and finally an explosion of the cell body like an over-inflated balloon (pointed with arrowhead in Figure 1E) in conjunction with PI uptake (Figure 1E). Scanning EM (SEM) revealed that necroptotic cells were round with bursting extensions (pointed with arrowhead in Figure 1G, TSZ). Intriguingly, pyroptotic cells displayed less swelling in comparison with necroptotic cells and produced multiple bubble-like protrusions (indicated by arrow in Figure $1 \mathrm{~F})$ before rupture of the plasma membrane (Figure $1 \mathrm{~F})$. Ultrastructures of control (DMSO) and LPS-treated cells were not different, whereas the bubble-like cell protrusions in LPS + Nig-treated cells progressed into protrusions with similar sizes of the apoptotic bodies (Figure 1G, LPS + Nig early), while the rest of the cells remained tightly attached to the culture slide followed by cytoplasm flattening (Figure 1G, LPS + Nig early and late). We designate the small protrusion bodies formed during pyroptosis pyroptotic bodies; and their nature is currently unknown. Corpses of pyroptotic cells looked like cabbage or fried egg with the cell's nucleus located in the center and above the main plane of the cell body (Figure 1G, LPS + Nig late). These observations reveal a clear difference in morphology between necroptosis and pyroptosis.

Similar to MLKL in necroptosis, GSDMD-N is translocated to the plasma membrane during pyroptosis

It has been demonstrated that execution of necroptosis is mediated by MLKL that has translocated to the plasma membrane [11-14]. By staining HA in RAW-asc cells expressing C-terminal HA-tagged MLKL (RAW-asc MLKL-HA), we detected the plasma membrane MLKL in necroptotic RAW-asc cells at early (PI-negative) and late (PI-positive) stages of necroptosis (Figure 2A). The translocation of MLKL to plasma membrane was further confirmed by colocalization analysis with plasma membrane marker Hras-RFP (Figure 2B). Notably, the discontinuous distribution of MLKL and Hras-RFP at the plasma membrane of necroptotic cells is consistent with the cell bursting observed under light microscope and EM (Figure 1E and 1G). To address the subcellular location of GSDMD when it executes pyroptosis, we expressed N-terminal HA-tagged GSDMD (HA-GSDMD) in $G s d m d^{-/}$RAW-asc cells. Immunostaining showed that HA-GSDMD was diffused uniformly in the cytosol 
A

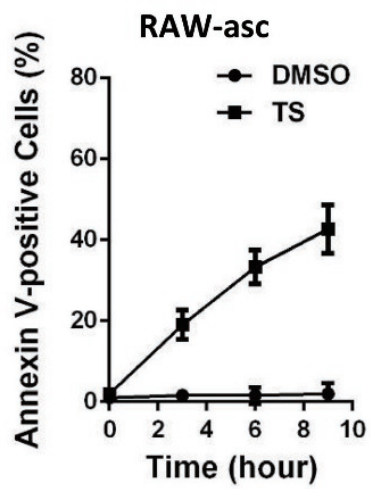

D

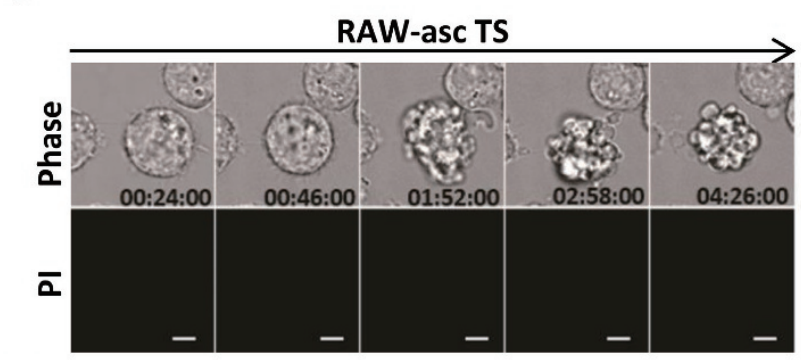

$\mathrm{E}$

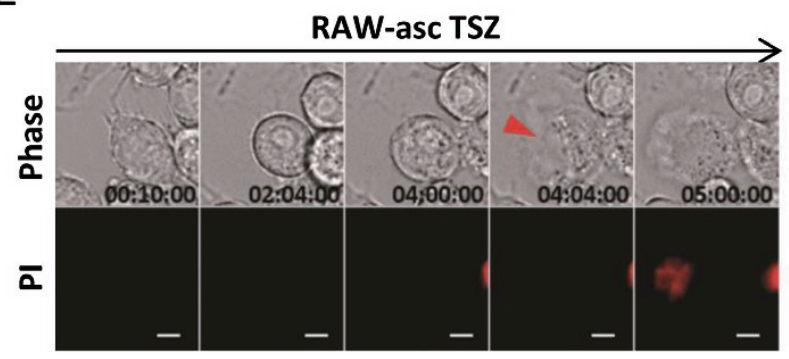

$\mathrm{F}$

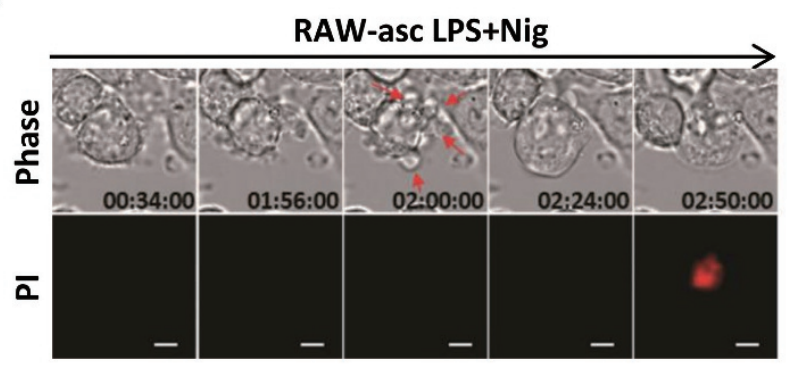

RAW-asc

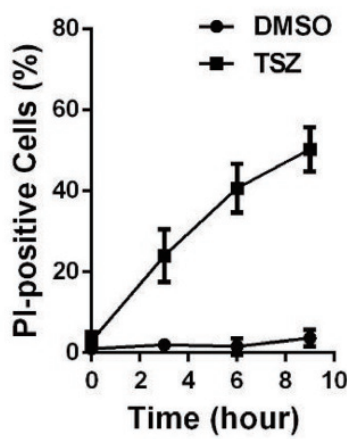

G
C

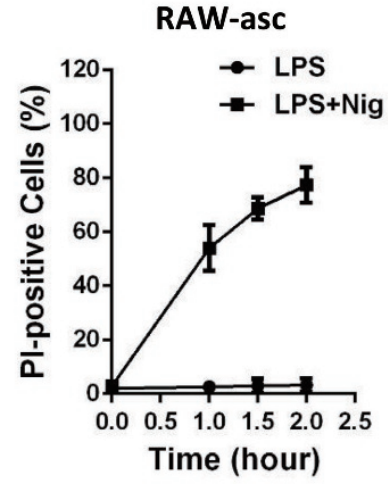

\section{RAW-asc}
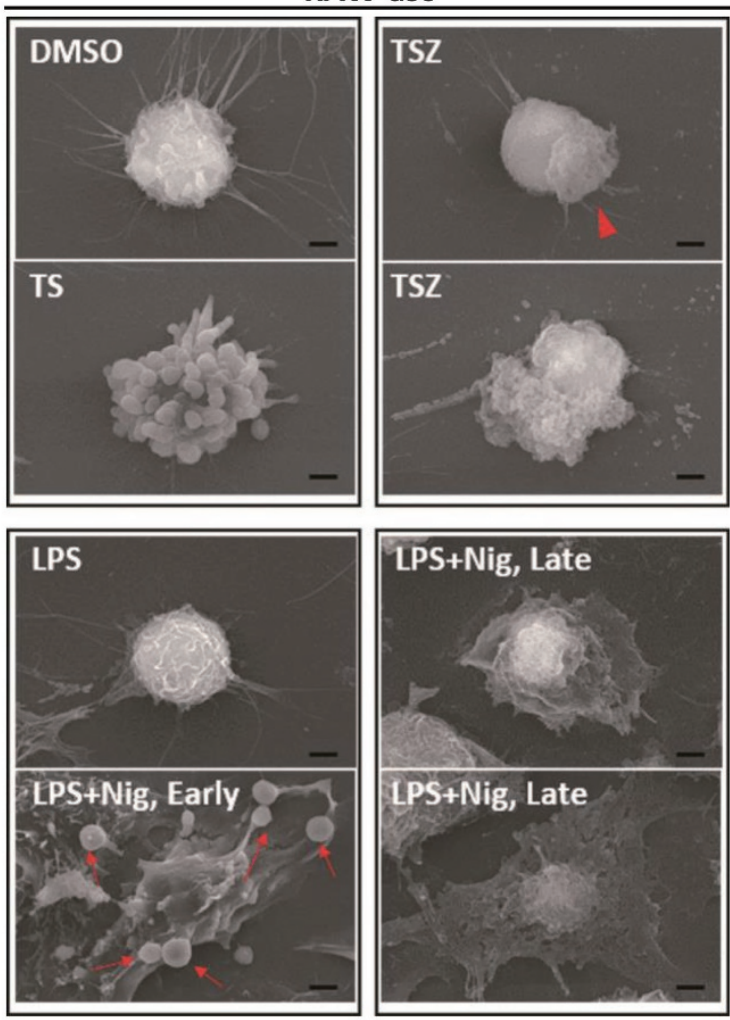

Figure 1 Pyroptotic and necroptotic cells have distinct morphological features. (A-C) Viabilities of RAW-asc cells treated with DMSO or TS (TNF and smac mimetic, A), or TSZ (TNF, smac mimetic and the caspase inhibitor Z-VAD, B), or primed with LPS for $4 \mathrm{~h}$ followed by nigericin (Nig) treatment or no treatment (C) for different periods of times as indicated. Apoptotic cells were identified by annexin $\mathrm{V}$ staining. Necroptosis and pyroptosis were determined by propidium iodide (PI) staining. Results shown are mean \pm SD from three independent experiments. The final concentrations of $10 \mathrm{ng} / \mathrm{ml} \mathrm{TNF}, 100 \mathrm{nM}$ smac mimetic, $20 \mu \mathrm{M} z-V A D, 1 \mu \mathrm{g} / \mathrm{ml}$ LPS and $10 \mu \mathrm{M}$ Nig were used here and in subsequent experiments unless otherwise stated. (D-F) Representative time-lapse images of RAW-asc cells treated as in A-C. Cell morphology was visualized by wide-field light microscopy (upper panel) and cell membrane integrity was monitored by PI uptake (lower panel). (G) Representative scanning electronic microscopy (SEM) images of RAW-asc cells treated as in A-C. Arrowhead indicates explosion of necroptotic cells and arrow points to bubbling of pyroptotic cells. Scale bar, $5 \mu \mathrm{m}$ (D-F) and $2 \mu \mathrm{m}$ (G). 


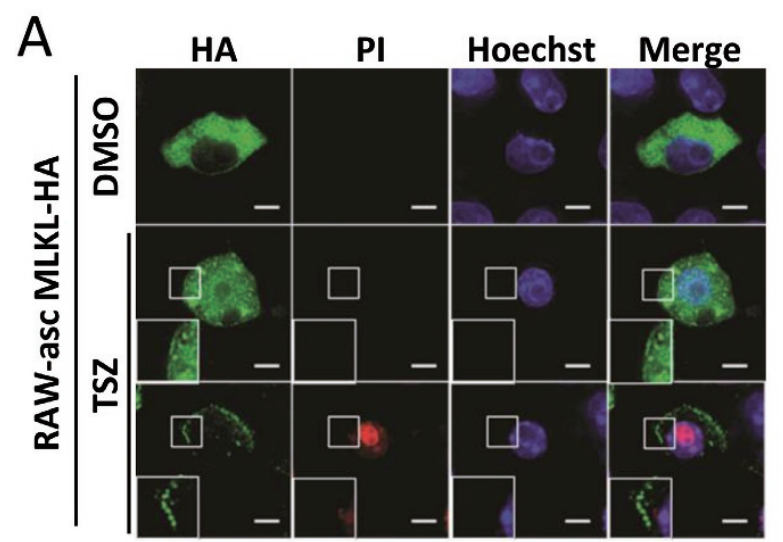

B

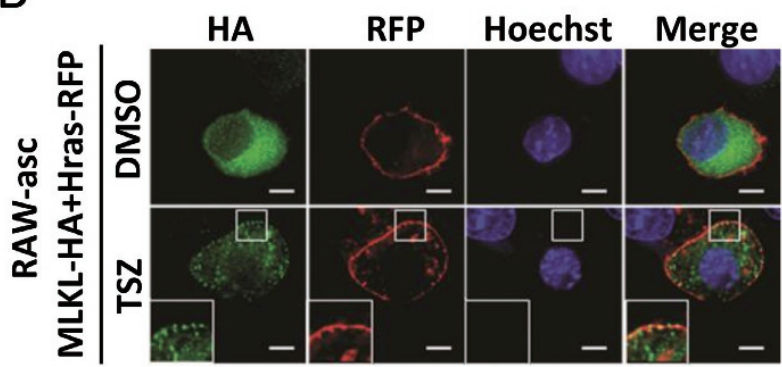

C

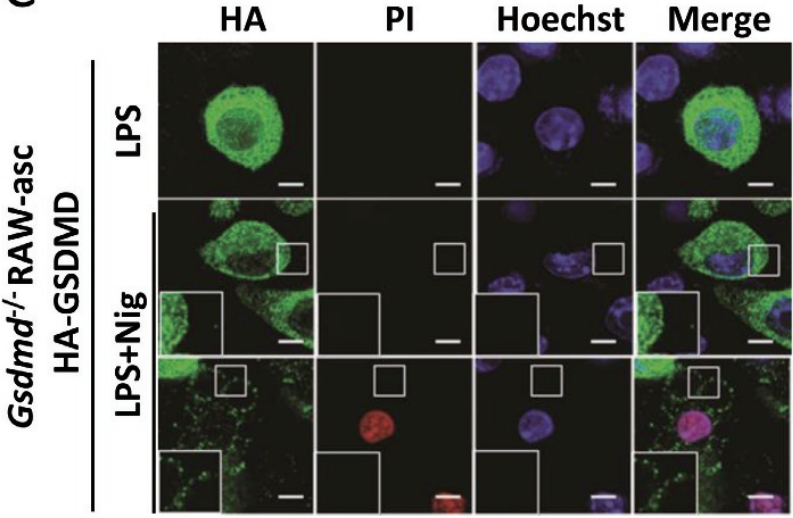

D

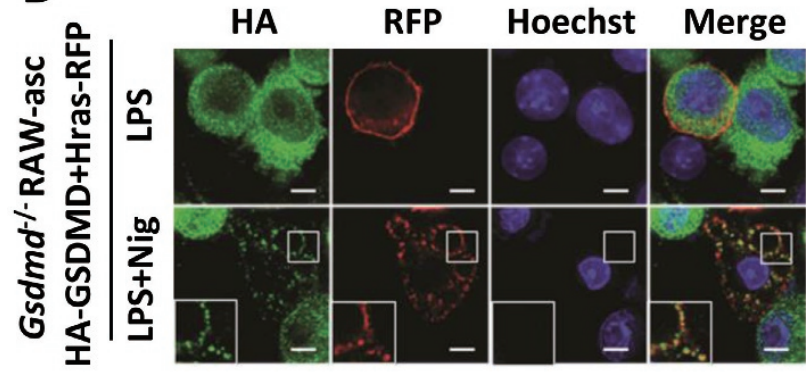

$\mathrm{E}$

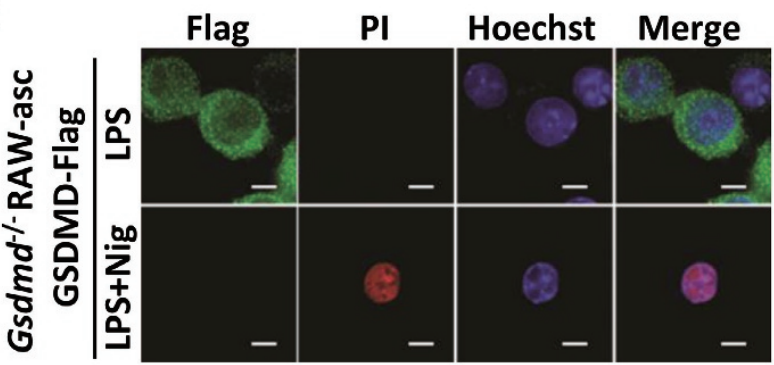

$\mathrm{F}$

Gsdmd/-RAW-asc HA-GSDMD

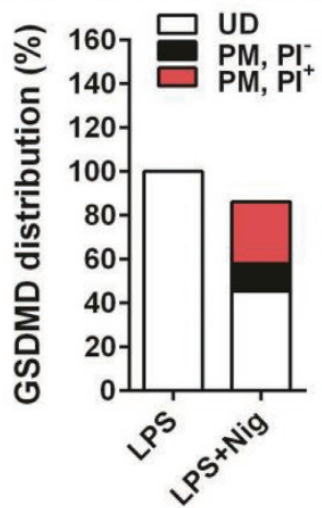

G

$$
\begin{aligned}
& \text { Triglyceride } O \quad O \mathrm{PI} \\
& \text { DAG } \bigcirc \quad 0 \text { Ptdins(4)P } \\
& \text { PA } \bigcirc \text { O } \operatorname{Ptdlns}(4,5) \mathbf{P}_{2} \\
& \text { PS } \bigcirc \quad \bigcirc \quad \text { Ptdins }(3,4,5) \mathbf{P}_{3} \\
& \text { PE } O \text { C Cholesterol } \\
& \text { PC } O \text { Sphingomyelin } \\
& \text { PG } O \text { O Sulfatide }
\end{aligned}
$$$$
\text { Cardiolipin } O \text { Blank }
$$

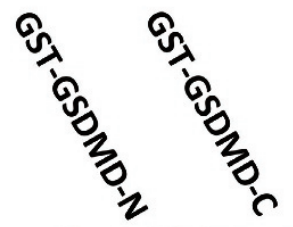

$\mathrm{H}$

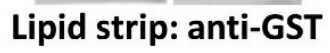

\section{RAW-asc}

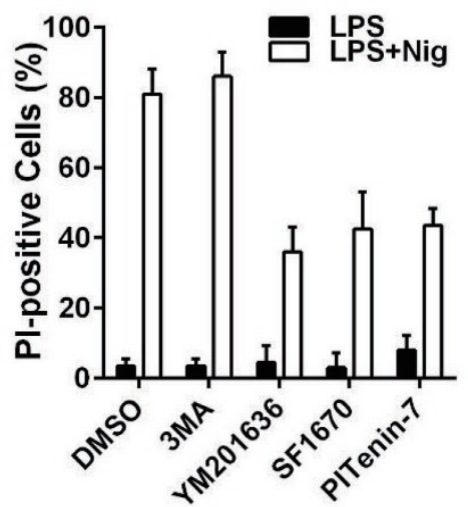

on the plasma membrane at early stage of pyroptosis (PI-negative, Figure 2C). We have shown in Figure 1G of cells treated with LPS (Figure 2C). When Nig was added, we observed an enriched HA-GSDMD staining 
Figure 2 GSDMD-N translocates to plasma membrane in pyroptotic cells. (A) Deconvolution microscopy of MLKL-HA-expressing RAW-asc cells treated with DMSO or TSZ for $5 \mathrm{~h}$. After treatment, cells were immunostained for HA and counterstained with Hoechst and PI. PI positive indicates dead cell. (B) Plasma membrane marker Hras-RFP was expressed and detected in MLKL-HA-expressing RAW-asc cells. Cells were treated and analyzed as in A. (C) Deconvolution microscopy of HA-GSDMD-reconstituted Gsdmd ${ }^{1-}$ RAW-asc cells primed with LPS for $4 \mathrm{~h}$ and subsequently treated with or without Nig for $2 \mathrm{~h}$. (D) The same as in C except that plasma membrane marker Hras-RFP was expressed and detected in HA-GSDMD-reconstituted RAW-asc cells. (E) Gsdmd ${ }^{-/}$RAW-asc cells were reconstituted with GSDMD-Flag, then treated and analyzed as in C. (F) Quantification of cells in C with uniformly diffused GSDMD (UD), plasma membrane enriched GSDMD plus PI-positive (PM, $\mathrm{PI}^{+}$) and plasma membrane enriched GSDMD plus PI-negative (PM, $\mathrm{PI}^{-}$). (G) Purified recombinant GST-GSDMD-N and GST-GSDMD-C were incubated with a general lipid strip, respectively. Protein-lipid binding assay was performed as described in the Material and Methods. (H) Raw-asc cells were primed with LPS for $4 \mathrm{~h}$ and then pretreated with $3 \mathrm{MA}$ (10 mM), YM201636 $(80 \mu \mathrm{M})$, SF1670 $(2 \mu \mathrm{M})$ or PITenin-7 $(20 \mu \mathrm{M})$ for $1 \mathrm{~h}$. Cells were then treated with Nig for $2 \mathrm{~h}$ and cell survival was determined by PI staining. Results shown are mean \pm SD representative of three independent experiments. Scale bar, 5 $\mu \mathrm{m}$.

that late-stage pyroptotic cells are flattened. The HA staining in the late-stage pyroptotic cells (PI-positive) was on membrane-like structure (Figure 2C) and we predict that this structure is a section view of the plasma membrane of cytoplasm-flattened pyroptotic cells. Indeed, in colocalization analysis HA-GSDMD and Hras-RFP exhibited similar distribution in pyroptotic cells, whereas Hras-RFP was separated from cytosolic HA-GSDMD in LPS-treated cells (Figure 2D). We also investigated the distribution of GSDMD C-terminal fragment (GSDMD-C) in pyroptotic cells using $G s d m d^{-/-}$ cells reconstituted with C-terminal Flag-tagged GSDMD (GSDMD-Flag, Figure 2E). In sharp contrast, GSDMDC-Flag was completely absent in dead cells (PI-positive), implying its release after plasma membrane breakage. This confirms that GSDMD-N but not GSDMD-C is translocated onto the plasma membrane. Further quantitation revealed that $12.5 \%$ of LPS + Nig-treated cells had GSDMD on the plasma membrane but were PI-negative ( $\left.\mathrm{PM}, \mathrm{PI}^{-}\right), 28.3 \%$ of cells had GSDMD on the plasma membrane and were PI-positive (PM, $\left.\mathrm{PI}^{+}\right)$, and $45.5 \%$ of cells had GSDMD uniformly distributed (UD; Figure 2F). Affinity to phospholipids is likely to be a property of MLKL that determines its plasma membrane interaction $[13,14,27]$. We tested whether GSDMD-N can interact with lipids by using lipid strips. Recombinant GST-GSDMD-N and GST-GSDMD-C were incubated individually with pre-loaded lipid strips. GST-GSDMD-N but not GST-GSDMD-C bound to phosphatidylinositol (4)-phosphate (PI4P), phosphatidylinositol (4,5)-biphosphate $\left(\mathrm{PI}(4,5) \mathrm{P}_{2}\right)$, and to a lesser extent phosphatidylinositol $(3,4,5)$-triphosphate $\left(\mathrm{PI}(3,4,5) \mathrm{P}_{3}\right)$ (Figure $\left.2 \mathrm{G}\right)$. Since GST-GSDMD-N was associated with a broad range of phosphatidylinositol phosphates (PIPs), we tested whether PIPs could play a role in pyroptosis. To address this, we used several chemical compounds that can affect the concentration of PIPs. PIKfyve inhibitor YM201636, PTEN inhibitor SF1670, and $\mathrm{PI}(3,4,5) \mathrm{P}_{3}$ antagonist PIT- enin-7 inhibited pyroptosis, whereas PI3K inhibitor 3MA had no effect (Figure 2H). In summary, we conclude that there is translocation of GSDMD-N to the plasma membrane during pyroptosis.

Artificially forced GSDMD-N interaction induces cell death with pyroptotic characteristics

Our previous study showed that ion influx occurs prior to plasma membrane rupture during necroptosis [11]. Since Nig is an ionophore derived from Streptomyces hygroscopicus which can transport several types of ions $[28,29]$, it would not be feasible if we want to dissect the molecular events leading to pyroptosis using the Nig induction model. Considering that translocation to the plasma membrane is required for both MLKL and GSDMD to mediate necrosis and forcing MLKL to dimerize using hormone-binding domain (HBD*)-mediated dimerization can mimic stimulus-induced MLKL activation of necroptosis, we tested whether the same method can be used to study GSDMD-mediated pyroptosis. We fused GSDMD-N and GSDMD-C with HBD*, and expressed them in Chinese hamster ovary (CHO) cells. HA tag was also fused to the C-terminus of GSDMD-N-HBD* and GSDMD-C-HBD* for immunostaining analysis. Induction of GSDMD-N-HBD* interaction by 4-hydroxytamoxifen (4-OHT) quickly triggered cell death in $\mathrm{CHO}$ cells (Figure $3 \mathrm{~A})$. As anticipated, the interaction of GSDMD-C-HBD* had no effect on cell viability (Figure 3A). Next, we performed time-lapse imaging to analyze 4-OHT-induced cell death in GSDMD-N-expressing $\mathrm{CHO}$ cells, and found these cells, like pyroptotic RAW-asc cells (Figure $1 \mathrm{~F}$ ), displayed multiple bubbles and subsequent rupture of the plasma membrane (Figure 3B). SEM revealed that the dying cells remained adhered to the culture surface and released several pyroptotic bodies (Figure 3C). Immunostaining of HA in GSDMD-N-HBD*-HA-expressing $\mathrm{CHO}$ cells showed that GSDMD-N was translocated upon 4-OHT treatment (Figure 3D) to the plasma mem- 
A

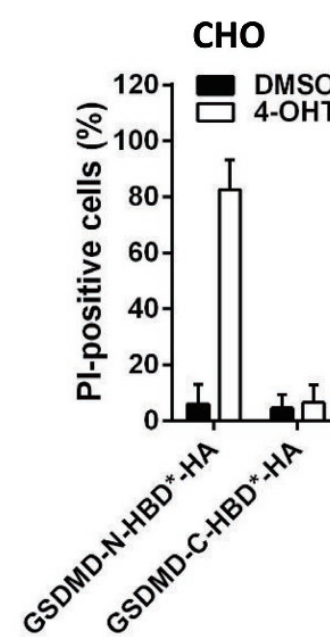

C

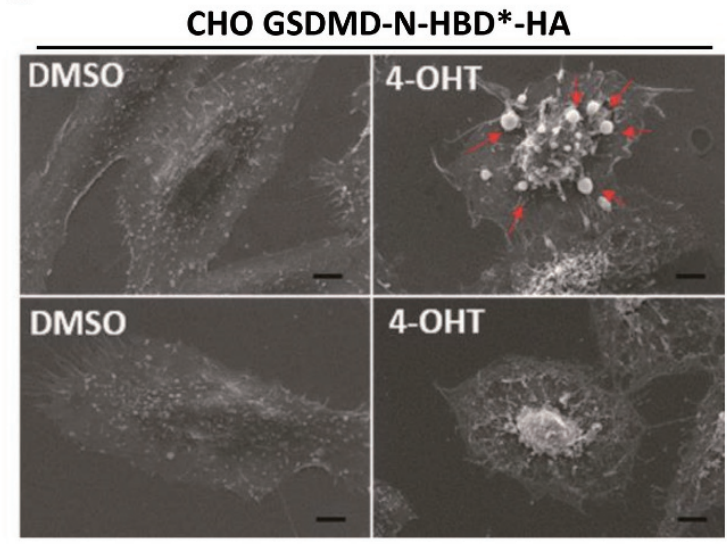

E

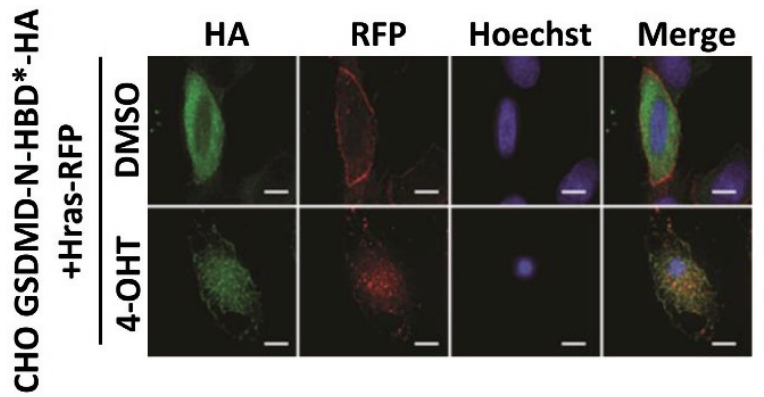

B

$\mathrm{F}$

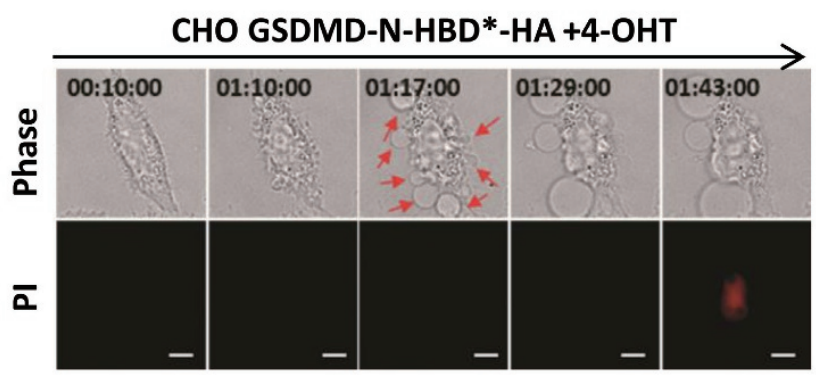

D

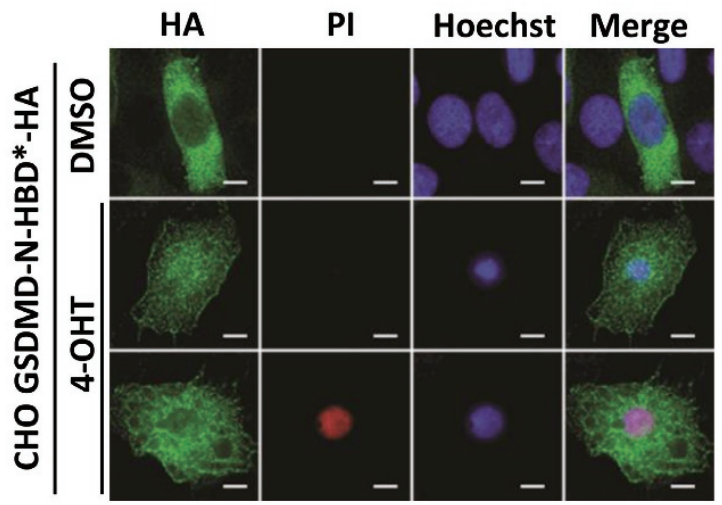

\section{CHO GSDMD-N-HBD*-HA}

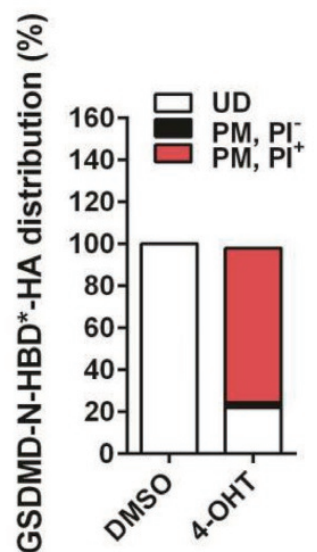

Figure 3 Forced interaction of GSDMD-N leads to pyroptosis. (A) GSDMD-N-HBD*-HA or GSDMD-C-HBD*-HA was expressed in $\mathrm{CHO}$ cells. These cells were treated with DMSO or $1 \mu \mathrm{M} 4-\mathrm{OHT}$ for $1 \mathrm{~h}$. Cell survival was determined by $\mathrm{PI}$ staining. Results shown are mean \pm SD from two independent experiments. The same concentration of 4-OHT was used in subsequent experiments unless otherwise stated. (B) Representative time-lapse imaging of GSDMD-N-HBD*-HA-expressing $\mathrm{CHO}$ cells treated with 4-OHT. Cell morphology was detected by wide-field light microscopy (upper panel) and cell membrane integrity was monitored by PI uptake (lower panel). Arrow indicates bubbling of pyroptotic cells. (C) Representative SEM images of GSDMD-N-HBD*-HA-expressing CHO cells treated with DMSO or 4-OHT for $1 \mathrm{~h}$. Arrow indicates bubbling of pyroptotic cells. (D) Deconvolution microscopy of GSDMD-N-HBD*-HA-expressing CHO cells treated with DMSO or 4-OHT for 1 h. PI was used to detect dead cells. (E) The same as D, except that plasma membrane marker Hras-RFP was expressed and detected in GSDMD-N-HBD*-HA-expressing CHO cells. (F) Quantification of cells in D with uniformly diffused GSDMD-NHBD*-HA (UD), plasma membrane enriched GSDMD-N-HBD*-HA plus PI-positive (PM, $\left.\mathrm{PI}^{+}\right)$and plasma membrane enriched GSDMD-N-HBD*-HA plus PI-negative (PM, $\left.\mathrm{PI}^{-}\right)$. Scale bar, $5 \mu \mathrm{m}(\mathbf{B}, \mathbf{D}$ and $\mathbf{E})$ and $2 \mu \mathrm{m}(\mathbf{C})$. 
brane labeled by plasma membrane marker Hras (Figure 3E). Quantitation of 4-OHT-treated cells revealed that $2.5 \%$ of cells were undergoing pyroptosis $\left(\mathrm{PM}, \mathrm{PI}^{-}\right)$, $73.6 \%$ of cells had completed pyroptosis $\left(\mathrm{PM}, \mathrm{PI}^{+}\right)$, and $21.8 \%$ of cells remained alive (UD) (Figure 3F). The same results were obtained when we used HeLa cells (Supplementary information, Figure S1A-S1E). These data together demonstrate that pyroptosis can be induced by forced interaction of GSDMD-N, and we can use this system to study pyroptosis.

GSDMD-N forms oligomers during pyroptosis

Oligomerization of MLKL is required for necropto-

A

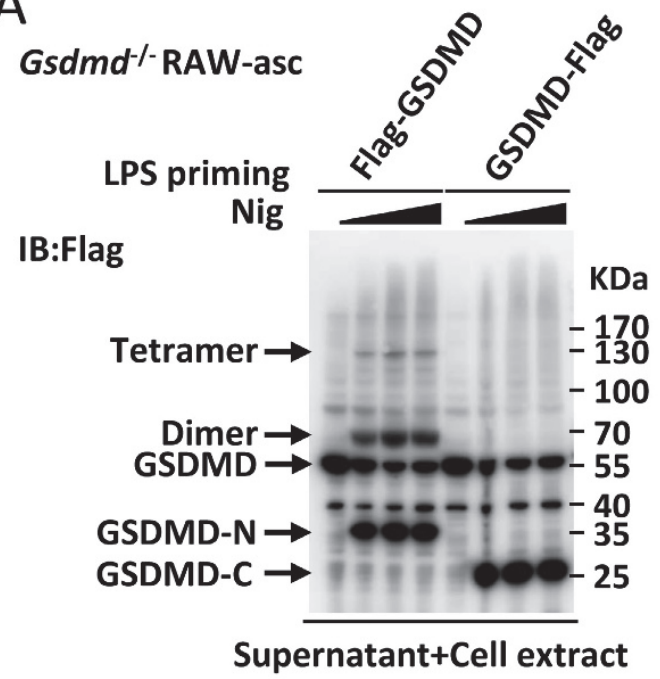

C

Gsdmd ${ }^{-/-R A W-a s c}$

Flag-GSDMD

Fraction $1 \begin{array}{lllllllllll}2 & 3 & 4 & 5 & 6 & 7 & 8 & 9 & 1011 & 1213141516171819202122\end{array}$

IB:Flag

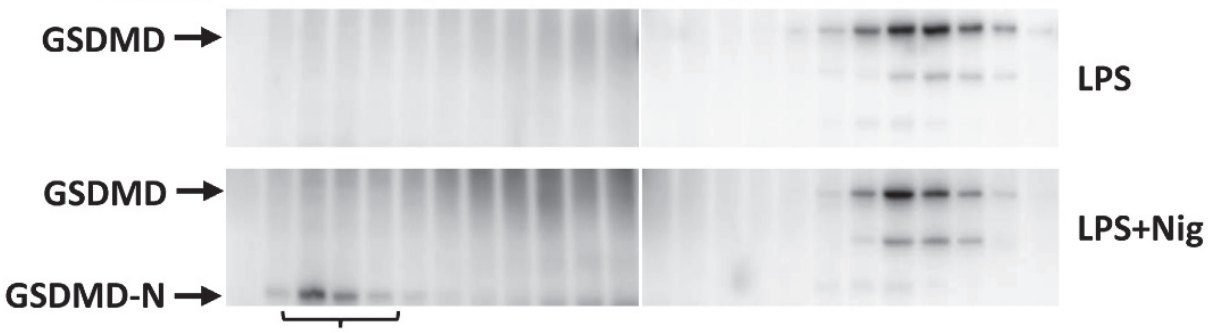

sis and we hypothesized that GSDMD may execute pyroptosis through a similar mechanism [11, 30, 31]. We therefore analyzed the oligomerization status of GSDMD using Gsdm $^{-/-}$RAW-asc cells reconstituted with Flag-GSDMD or GSDMD-Flag (Figure 4A). LPS + Nig-induced cleavage of Flag-GSDMD and GSDMD-Flag occurred at an equivalent rate as we detected approximately equal amount of Flag-GSDMD-N and GSDMD-C-Flag in Flag-GSDMD- and GSDMD-Flag-reconstituted cells respectively. The oligomers (dimers and tetramers) were detected only in Flag-GSDMD-reconstituted cells, indicating that GSDMD-N but not GSDMD-C formed oligomers. Disuccinimidyl su-

B

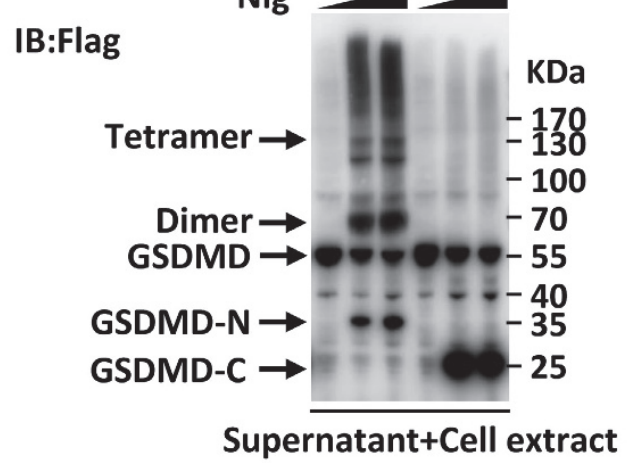

Figure 4 GSDMD-N but not GSDMD-C forms oligomer during pyroptosis. (A) Gsdmd ${ }^{-1}$ RAW-asc cells were reconstituted with Flag-GSDMD and GSDMD-Flag respectively, and then primed with LPS for $4 \mathrm{~h}$ followed by Nig stimulation for 0 min, 30 $\mathrm{min}, 60 \mathrm{~min}$ and $120 \mathrm{~min}$. The culture supernatants together with their corresponding cell extracts were resolved by $4 \%-12 \%$ gradient SDS-PAGE. Immunoblotting of Flag was performed. (B) The same cells were treated as in A. The culture supernatants together with their corresponding cell extracts were analyzed after DSS crosslinking. (C) Flag-GSDMD-reconstituted cells were primed with LPS followed by Nig stimulation or no stimulation. Lysates of these cells were fractionated by gel-filtration chromatography (Superose 6, fractions sizes at top) and followed by immunoblot analysis. 
A

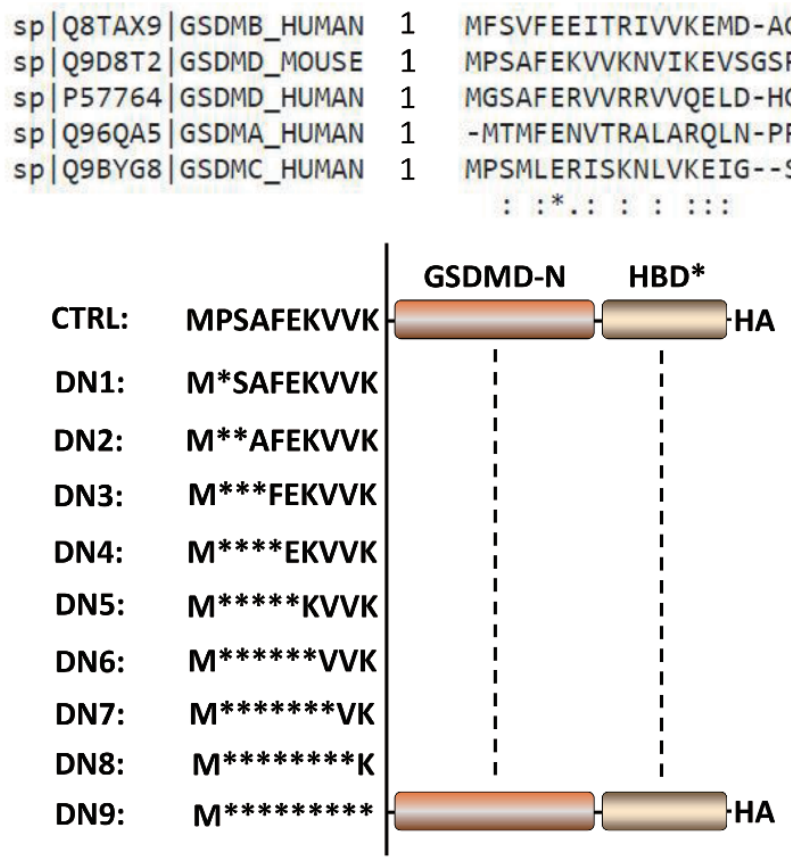

D

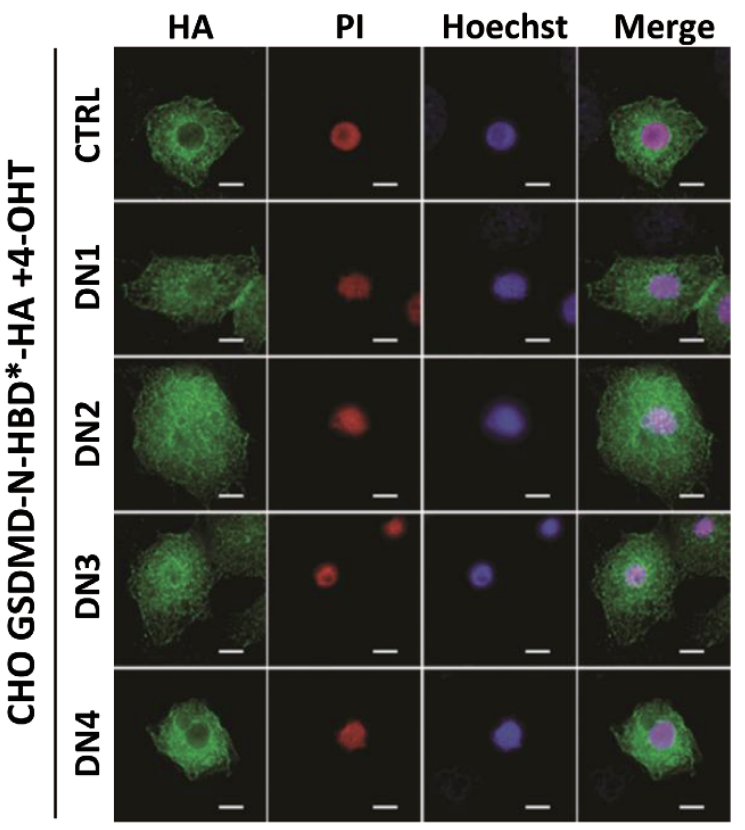

B

C
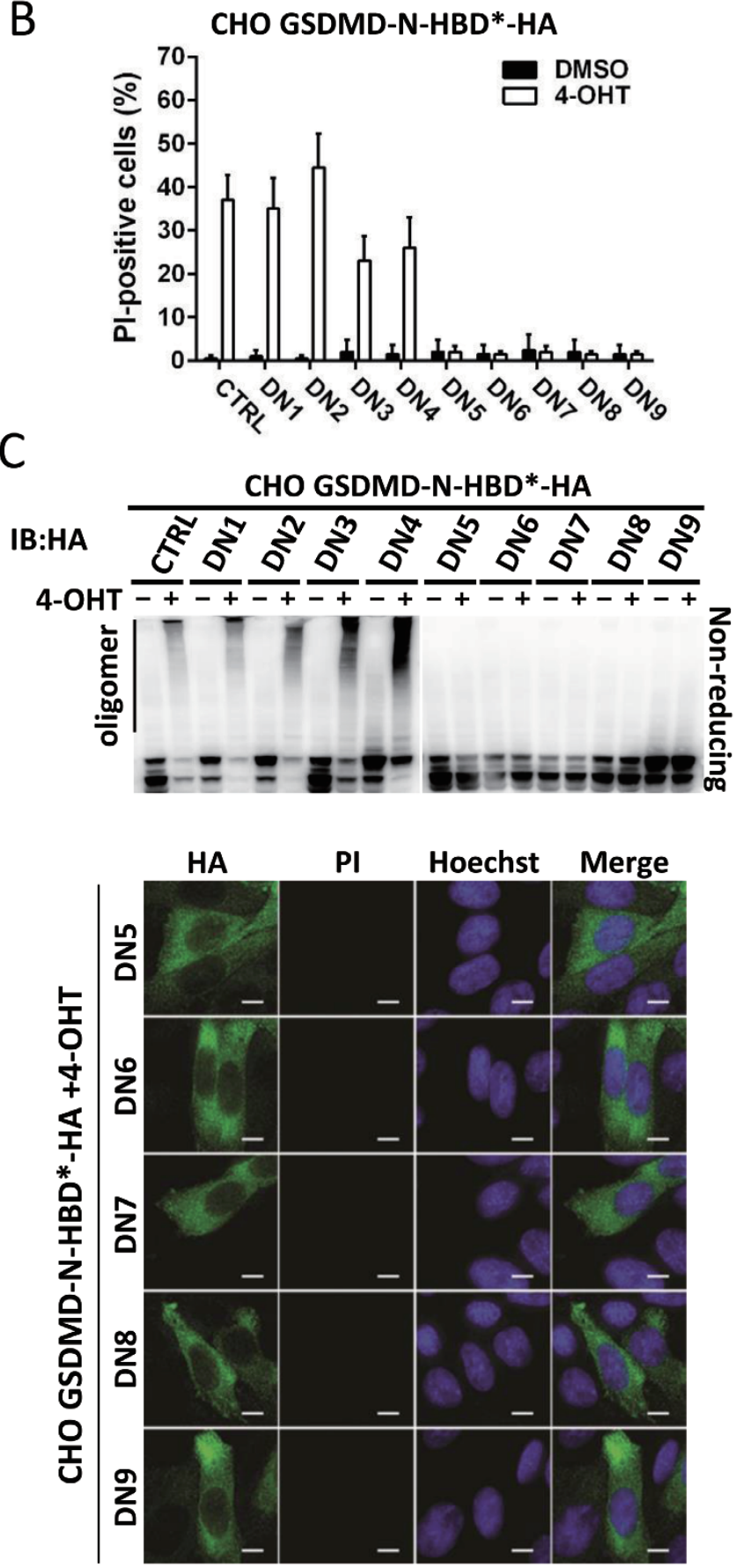

Figure $5 \mathrm{~N}$-terminal five amino acids are indispensable for GSDMD-N to form oligomers and trigger pyroptosis. (A) Sequence alignment of N-terminal 20 amino acids of human GSDMA, GSDMB, GSDMC, GSDMD and mouse GSDMD (upper panel), and schematic representation of N-terminal deletions of GSDMD-N fused with HBD* (lower panel). (B) Viabilities of $\mathrm{CHO}$ cells expressing different GSDMD-N-HBD*-HA deletion mutants after stimulation with DMSO or 4-OHT for 0.5 h. Data represented the mean \pm SD of three independent experiments. (C) Immunoblot analysis of lysates of $\mathrm{CHO}$ cells in $\mathbf{B}$ resolved by $4 \%-12 \%$ gradient SDS-PAGE under non-reducing condition. (D) Deconvolution microscopy of $\mathrm{CHO}$ cells expressing different GSDMD-N-HBD*-HA deletion mutants treated with 4-OHT for $1 \mathrm{~h}$. After treatment, cells were immunostained for HA and counterstained with Hoechst and PI. PI positive indicates dead cell. Scale bar, $5 \mu \mathrm{m}$. 
berate (DSS) crosslinking massively increased the size of GSDMD oligomers, suggesting the formation of a higher-order GSDMD complex (Figure 4B). We further used gel-filtration chromatography to fractionate the cell extract and found high molecular weight GSDMD-N complex in LPS + Nig-treated but not LPS-treated Flag-GSDMD-reconstituted RAW-asc cells (Figure 4C). We also expressed and purified GSDMD-N and GSDMD-C and found recombinant GST-GSDMD-N formed oligomers whereas GSDMD-C-His existed as monomers (Supplementary information, Figure S2A and S2B). These data together demonstrate that GSDMD-N forms oligomers during pyroptosis.

$N$-terminal five amino acids are indispensable for GSD$M D-N$ to form oligomers

When generating GSDMD-reconstituted RAW-asc cells, we noticed that the N-terminal $3 \times$ Flag tag but not $1 \times$ Flag tag abolished GSDMD oligomerization and GSDMD-mediated cell death, suggesting that N-terminal structure of GSDMD is important for its function (Supplementary information, Figure S3A and S3B). The first $15 \mathrm{~N}$-terminal amino acids are conserved in human and mouse gasdermin family (Figure 5A, upper panel) and were resolved as an $\alpha$-helix structure in a recent study [24]. We removed the N-terminal amino acids one by one and fused the rest part of GSDMD-N with HBD* and termed these mutants as GSDMD-N-HBD*-HA-DN1 to DN9 corresponding to the number of amino acids deleted (Figure 5A, lower panel). After introducing each of these mutants into $\mathrm{CHO}$ cells, we challenged the cells with 4-OHT and found that mutants missing more than five amino acids abolished GSDMD-N-HBD*-mediated cell death (Figure 5B) and GSDMD-N oligomerization (Figure $5 \mathrm{C}$ ), suggesting that oligomerization of GSDMD-N links to its ability to trigger pyroptosis. Immunostaining revealed that GSDMD-N-HBD*-HA-DN1 to DN4 but not DN5-DN9 behaved the same as wild-type GSDMDN-HBD*-HA (Figure 5D and Supplementary information, Figure S3C). Thus, the $\mathrm{N}$-terminal first $\alpha$-helix is important for GSDMD-N oligomerization.

Oligomerization of GSDMD-N is likely to initiate cell pyroptosis by forming non-selective pores in the plasma membrane

We next took advantage of 4-OHT-induced cell pyroptosis to analyze the change of intracellular ion homeostasis during pyroptosis. Sodium indicator ANG-2, potassium indicator APG-2, and calcium indicator Fluo4 were used in our experiments [32-34]. The intensity of ANG-2 increased initially then suddenly dropped when plasma membrane rupture occurred as indicated by PI uptake in GSDMD-N-HBD*-HA-expressing CHO cells (Figure 6A). Similar phenomenon was observed when Fluo-4 was used (Figure 6B). APG-2 showed a slightly different pattern of change after 4-OHT treatment, which underwent a slow reduction first and then suddenly dropped immediately before PI uptake (Figure 6C). This is most likely due to a higher intracellular potassium concentration than the medium [35]. In contrast, none of these phenomena was observed in $\mathrm{CHO}$ cells expressing GSDMD-C-HBD*-HA (Supplementary information, Figure S4A-S4C). The ion influx/efflux we observed in pyroptotic cells can be simply interpreted as a consequence of GSDMD-N-formed nonselective pores in the plasma membrane which dissipated cellular ionic gradients.

It was proposed previously that pyroptosis is mediated by pores in plasma membrane because pyroptosis can be inhibited by polyethylene glycol (PEG) of certain size [23]. We treated GSDMD-N-expressing CHO cells with 4-OHT diluted in PBS or PBS containing PEG of different diameters. In comparison with the cells cultured in PBS, cell death was partially prevented in PBS containing PEG1450 and PEG4000 and completely blocked in PBS containing PEG8000, but was not affected in PBS containing PEG400 (Figure 6D), consistent with published data that large size PEG can block the pores. Importantly, protection conferred by PEG4000 was abolished by changing buffer to PBS after the activation of pyroptotic pathway (Figure 6E). Collectively, these data indicate that plasma membrane-bound GSDMD-N forms non-selective ion-permeable pores, which disrupt plasma membrane and eventually lead to cell lysis.

\section{Discussion}

It is generally accepted that apoptosis is a safe death and necrosis causes inflammation [36]. In this study, we performed a comparative study of two programmed necrosis, necroptosis and pyroptotsis. We show that necroptotic cell death is more like a process of cell explosion and pyroptosis undergoes cytoplasm flattening caused by plasma membrane leakage. Although both necroptosis and pyroptosis are executed by altering plasma membrane permeability, the former exhibits ion selection but the latter has no selectivity. Because of the non-selectivity of GSDMD-formed pores, there should not be a substantial increase in intracellular osmolarity, which explains why pyroptotic cells do not swell and burst. Different from necroptoic cells, pyroptotic cells generate pyroptotic bodies with a size similar to apoptotic bodies. The differences in morphology between necroptosis and pyroptosis are presumably determined by the distinct mechanisms leading to the two forms of cell death. 
A

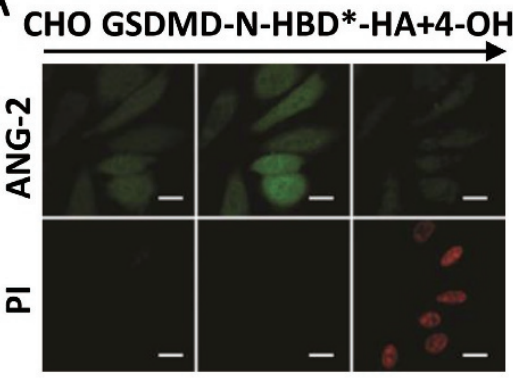

B

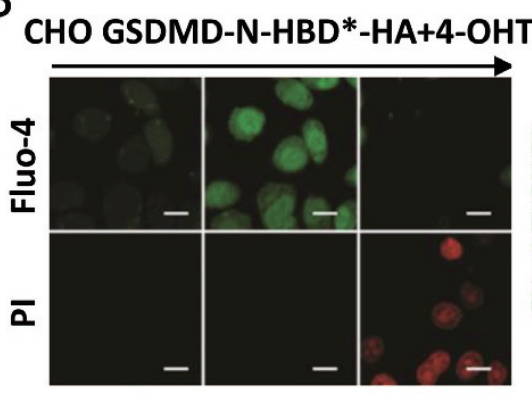

C

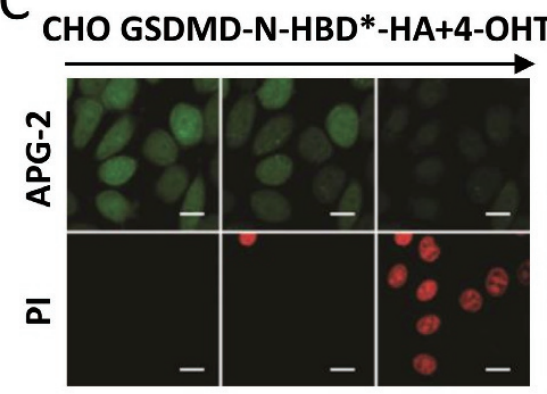

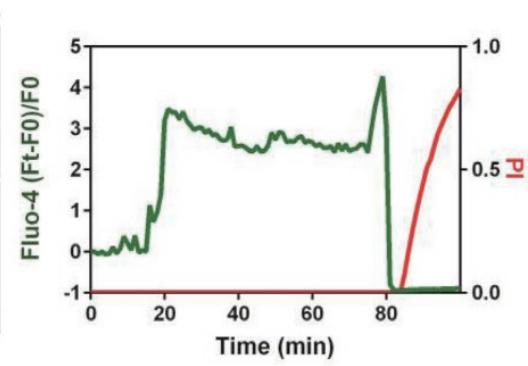

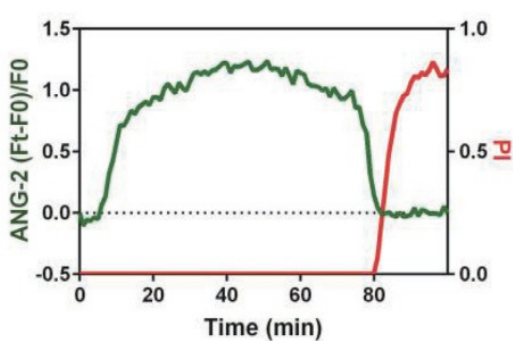

HT

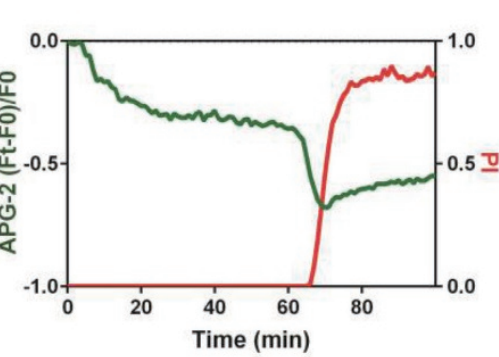

D

CHO GSDMD-N-HBD*-HA

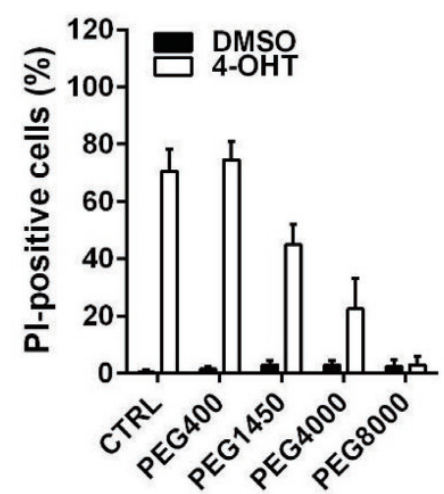

$\mathrm{E}$

CHO GSDMD-N-HBD*-HA

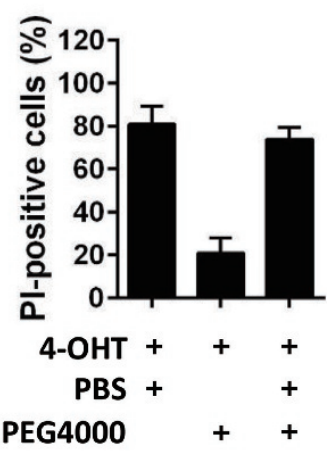

Figure 6 GSDMD-N non-selectively increase plasma membrane permeability. (A-C) Intracellular ion concentration were monitored in GSDMD-N-HBD*-HA-expressing CHO cells treated with 4-OHT. Sodium indicator (ANG-2, A), calcium indicator (Fluo-4, B) and potassium indicator (APG-2, C) were used together with PI. For each ion indicator, a series of representative time-lapse images were shown in the left panel and relative intensity change of ion indicator and $\mathrm{PI}$ fluorescence in one representative cell was graphed over time in the right panel. (D) GSDMD-N-HBD*-HA-expressing CHO cells were treated with 4-OHT for $0.5 \mathrm{~h}$ in PBS (CTRL) or PBS containing $30 \mathrm{mM}$ PEG400, PEG1450, PEG4000 or PEG8000. Cells were stained by $\mathrm{PI}$ and analyzed under microscope. (E) GSDMD-N-HBD*-HA-expressing CHO cells were treated with 4-OHT in PBS or in PEG4000 PBS for $0.5 \mathrm{~h}$, or were treated with 4-OHT in PEG4000 PBS for $0.5 \mathrm{~h}$ and then were changed into PBS for another 0.5 h. Cells were stained with PI and analyzed under microscope. Results shown are mean \pm SD from two independent experiments. Scale bar, $10 \mu \mathrm{m}$.

Both necroptosis and pyroptosis require oligomerization and translocation of their executors to the plasma membrane. In necroptosis the initiation of MLKL oligomerization and plasma membrane translocation is mediated by phosphorylation [15, 37, 38], whereas GSDMD oligomerization and plasma translocation is initiated by caspase-mediated removal of C-terminal inhibitory domain. Monomer-to-oligomer transition is a general strategy applied in many signaling pathways. For instance, conversion of MAVS to prion-like aggregates functions as a platform to activate downstream IRF3 upon viral infection [39]. We found GSDMD-N forms dimer, tetramer and oligomer. It seems that there are multiple steps in the oligomerization process before the formation of higher-order complex. GSDMD-N oligomerization may occur prior to its migration to the plasma membrane, and this event precedes cell death since a fraction of cells displayed plasma membrane signal of GSDMD-N 
but were PI-negative. According to the crystal structure of GSDMD, first $15 \mathrm{~N}$-terminal amino acids form an $\alpha$-helix which plays an important role in autoinhibition of GSDMD [24]. Since N-terminal five amino acid deletion abolished GSDMD-N oligomerization and GSDMD-N-HBD*-mediated cell death, the N-terminal $\alpha$-helix should also be required for GSDMD oligomerization and function in pyroptosis. Interestingly, the N-terminal ten amino acids in MLKL are also in an $\alpha$-helix and are also required for MLKL oligomerization and function in necroptosis [11].

Both MLKL and GSDMD have been studied using artificial liposomes. MLKL acts as selective cation channel in liposomes [40]; and GSDMD-N forms EM-visible pores in the membrane of liposomes [24-26]. That pores with diameters ranging from 1.1 to $2.4 \mathrm{~nm}$ mediate pyroptosis has been proposed previously based on PEG-mediated inhibition of pyroptosis. EM measurement in recent studies revealed that pores formed by GSDMD-N have an inner diameter of 10-15 nm [24-26]. Our data are consistent with these published results that GSDMD-N forms pores in the plasma membrane, we further demonstrate that GSDMD-N-based pores are nonselective to different ions. $10-15 \mathrm{~nm}$ pores should be much larger than MLKL channels and a large number of such pores could conceivably destruct the plasma membrane, thereby promoting the formation of pyroptotic bodies and inducing plasma membrane disruption.

Changes of cell volume often accompany cell death and are typically driven by ion-involved osmotic pressure [41]. Efflux of intracellular potassium and chlorine is thought to be required for cell shrinkage, a phenomenon frequently observed in apoptotic cells [42]. MLKL on the plasma membrane results in overload of selected ions which drives water influx until cells burst. By contrast, pores formed by GSDMD lack selectivity and dissipate natural ionic gradients. However, we still observed slight cell swelling during pyroptosis, which may be caused by water influx driven by intracellular non-ionic osmolytes.

In summary, our study reveals substantial morphological differences between pyroptosis and necroptosis, and provides mechanistic explanations for these differences. The discovery of pyroptotic body is unexpected and warrants further studies to address the mechanistic links with other aspects of the pyroptotic process. As it is well known that pyroptosis is usually accompanied by the secretion of leaderless cytokine IL-1 $\beta$ [43], further investigation on GSDMD-mediated action in the plasma membrane will no doubt enhance our knowledge not only in inflammatory cell death but also in its role in the secretion of leaderless cytokines.

\section{Materials and Methods}

\section{Cell culture}

RAW 264.7, HEK293T, HeLa and CHO cells were obtained from ATCC. Gsdmd $d^{/-}$RAW-asc cells were generated as previously described [22]. All cells were cultured in Dulbecco's modified Eagle's medium (DMEM) supplemented with $10 \%$ fetal bovine serum, $2 \mathrm{mM} \mathrm{L}$-glutamine, $100 \mathrm{IU}$ penicillin, and $100 \mathrm{mg} / \mathrm{ml}$ streptomycin at $37{ }^{\circ} \mathrm{C}$ in a humidified incubator containing $5 \%$ $\mathrm{CO}_{2}$.

\section{DNA constructs and lentivirus infection}

The human and mouse GSDMD cDNAs were PCR amplified from a reverse-transcribed cDNA library derived from HeLa and RAW 264.7 cells, respectively. Full-length or truncated cDNAs of GSDMD were cloned into BamHI and XhoI sites of the lentiviral vector $\mathrm{pBOB}$ using the Exo III-assisted ligase-free cloning method. pBOB-Hras-RFP was generated as previously reported [27]. All of plasmid constructs were confirmed by DNA sequencing. Stable cell lines were generated by lentivirus infection. Recombinant lentiviruses were produced by HEK293T cells co-transfected with a $\mathrm{pBOB}$ construct encoding a given cDNA and packaging plasmids by the calcium phosphate precipitation method. Viral supernatant was collected 36-48 h later and was used to infect cells. $10 \mu \mathrm{g} / \mathrm{ml}$ of polybrene was included in the infection to increase the infection efficiency.

\section{Reagents and antibodies}

Mouse TNF was purchased from eBioscience. Nigericin (Nig) and Hoechst 33342 were obtained from Invitrogen. Smac mimetic was from APExBIO. Ultrapure lipopolysaccharides (LPS), 4-hydroxytamoxifen (4-OHT), propidium iodide (PI), SF1670 and 3-methyladenine (3MA) were purchased from Sigma-Aldrich. YM201636 was provided by Invivogen. PITenin-7 and z-VAD were acquired from Calbiochem. All ion indicators including Asante NaTRIUM Green-2 (ANG-2) and Asante Potassium Green-2 (APG-2) were provided by TEFLabs, except that Fluo-4 was from Invitrogen. Antibodies were from commercial sources: Rabbit anti-Flag antibody (14793S, Cell Signaling), Mouse anti-HA antibody (sc-7392, Santa Cruz) and Mouse anti-GST antibody (M20007, Abmart).

\section{Immunoblotting and crosslinking}

Cell lysates were prepared in lysis buffer $(150 \mathrm{mM}$ Tris$\mathrm{HCl}, 3 \% \mathrm{SDS}, 0.15 \%$ bromophenol blue and $15 \%$ glycerol, $\mathrm{pH}$ 6.8 ) with or without $\beta$-mercaptoethanol. For crosslinking, cells were rinsed once with PBS and lysed in a buffer containing 12.5 mM HEPES pH 7.5, $30 \mathrm{mM} \mathrm{NaCl}, 90 \mathrm{mM}$ NaSCN, 1\% NP-40 and $0.5 \mathrm{mM}$ DSS (Thermo Fisher). Crosslinking reactions were maintained for $30 \mathrm{~min}$ at room temperature and then quenched by adding 1/10 v/v $1 \mathrm{M}$ Tris- $\mathrm{HCl}(\mathrm{pH}$ 7.4). In some Western blotting experiments, supernatants were also collected and precipitated with $20 \%$ trichloroacetic acid (TCA) $(20 \%$ final volume, $10 \mathrm{~min}$ at $\left.4{ }^{\circ} \mathrm{C}\right)$. Protein pellet was obtained by centrifugation $(17000 \times$ $g, 5 \mathrm{~min}$ at $4{ }^{\circ} \mathrm{C}$ ) and washed with ice-cold acetone for further analysis. Samples were subjected to electrophoresis using NuPage 4\%-12\% Bis-Tris gel (Life Technologies) and then transferred to polyvinylidene difluoride (PVDF) membranes by electroblotting. Immunoreactive proteins were visualized with ECL method 
(Pierce) and imaged by a Fujifilm LAS-4000 imager.

\section{Cell viability assay}

To trigger pyroptosis, Raw-asc cells were pretreated with $1 \mu \mathrm{g} /$ $\mathrm{ml}$ LPS for $4 \mathrm{~h}$ followed by $10 \mu \mathrm{M}$ Nig for the indicated time periods. Apoptosis and necroptosis were induced by treating RAW-asc cells with TS and TSZ, respectively. GSDMD-N-HBD*-HA-reconstituted cells were added with $1 \mu \mathrm{M}$ 4-OHT to induce cell pyroptosis. To test the effect of chemical compounds on cell pyroptosis, LPS-primed Raw-asc cells were pretreated individually with compounds including 3MA (10 mM), SF1670 (2 $\mu \mathrm{M})$, YM201636 $(80 \mu \mathrm{M})$ and PITenin-7 $(40 \mu \mathrm{M})$ for $1 \mathrm{~h}$ and then stimulated with $10 \mu \mathrm{M}$ Nig. Apoptotic cells were identified by annexin $\mathrm{V}$ staining (Biovision) while necroptosis and pyroptosis were determined by PI staining.

\section{Protein purification and gel filtration chromatography}

The constructs of GST-GSDMD-N, GST-GSDMD-C and GSDMD-C-His were transformed into E. coli strain BL21 (DE3). The expression of all proteins was induced overnight with $0.2 \mathrm{mM}$ isopropyl- $\beta$-Dthiogalactopyranoside (IPTG) at $20{ }^{\circ} \mathrm{C}$ except the temperature used for GST-GSDMD-N was $15^{\circ} \mathrm{C}$. Cells were collected and suspended in lysis buffer $(20 \mathrm{mM}$ Tris- $\mathrm{HCl} \mathrm{pH} 8.0,100$ $\mathrm{mM} \mathrm{NaCl}$ and $5 \mathrm{mM}$ 2-mercaptoethanol). GST-tagged and Histagged proteins were first purified with $\mathrm{Ni}^{2+}$-NTA (Qiagen) and glutathione beads (GE Healthcare), respectively. All proteins were further purified by Superpose 6 or Superdex 75 gel filtration and dialyzed against storage buffer $(20 \mathrm{mM}$ Tris- $\mathrm{HCl} \mathrm{pH}$ 8.0, $150 \mathrm{mM}$ $\mathrm{NaCl}, 3 \mathrm{mM}$ dithiothreitol (DTT)). Besides, Flag-GSDMD-reconstituted RAW-asc cells were incubated with NP-40 lysis buffer and then sonicated gently. Soluble lysate $(0.6 \mathrm{mg}$ of total protein) was run on a gel-filtration chromatography column (Superose 6, GE Healthcare) in NP-40 lysis buffer with $1 \mathrm{mM}$ DTT.

\section{PIP binding assay}

Recombinant GST-GSDMD-N and GST-GSDMD-C were diluted with blocking buffer (TBST with 3\% fatty acid free BSA). The protein-lipid interactions were analyzed with commercial lipid strips (Echelon Biosciences) according to the manufacturer's instructions. Briefly, membrane lipid strip was incubated with blocking buffer for $1 \mathrm{~h}$ at room temperature. A total of $5 \mu \mathrm{g}$ recombinant protein was incubated on lipid strip for $2 \mathrm{~h}$ at room temperature in blocking buffer. Binding of the proteins to the lipids was revealed by immunoblotting using mouse anti-GST.

\section{Scanning electron microscopy}

Cells were fixed with $2.5 \%$ glutaraldehyde overnight, and then rinsed with PBS three times. Sample were dehydrated through a graded series of ethanol $(30,50,70,95$ and 100\%) and dried by tertiary butanol method. Dried specimens were sputter coated with gold-palladium and imaged with a JEOL JSM 66390LV field emission scanning electron microscope operating at $10 \mathrm{kV}$.

\section{Immunofluorescence imaging}

For fixed cell imaging, cells were grown on \#1.5 coverslips (Nest) pre-coated with poly-L-lysine (Sigma-Aldrich). In some experiments, cells were stained first with PI to represent the dead cells. Cells were washed once with PBS followed by fixation with freshly prepared $4 \%$ paraformaldehyde (PFA) in PBS for 15 min at room temperature. The fixed cells were then permeabilized in $0.2 \%$ Triton X-100/PBS, blocked with 3\% BSA in PBS and stained with primary antibody and secondary antibody as indicated. Cells were counterstained with Hoechst to visualize nuclei. Representative deconvolution images were generated on a Delta-Vision OMX v4 (GE Healthcare) using a 60×/1.42 NA objective lens (Olympus). Immersion oil with a refractive index ranging from 1.514 to 1.518 was used depending on the fluorochromes labeled. Seventy optical sections were collected with $0.25 \mu \mathrm{m}$ spacing in each data set. Image deconvolution was performed using softWoRx software (GE Healthcare) with default setting.

For time-lapse microscopy, cells were plated onto a $35-\mathrm{mm}$ glass bottom dish (Nest). To monitor the intracellular ion concentration, CHO cells were loaded with sodium indicator ANG-2, potassium indicator APG-2 and calcium indicator Fluo-4 respectively according to the manufacturer's protocol. PI (5 $\mathrm{ng} / \mathrm{ml})$ was added to medium for monitoring cell membrane integrity. Imaging was carried out using Zeiss LSM 780 with a $63 \times / 1.49$ NA oil objective. Unprocessed images were analyzed by ImageJ software. To estimate changes in ion indicators fluorescence over time in confocal images, individual cells were selected manually and fluorescence values measured over the entire stack. For each condition, at least three independent experiments were run and analyzed.

\section{Acknowledgments}

We thank Luming Yao and Caiming Wu for help with electron microscopy. This work was supported by the National Basic Research Program of China (973 Program; 2015CB553800, 2013CB944903, 2014CB541804), the National Natural Science Foundation of China (91029304, 31420103910 and 31330047), the 111 Project (B12001), the National Science Foundation of China for Fostering Talents in Basic Research (J1310027) and the Science and Technology Foundation of Xiamen (No. 3502Z20130027).

\section{Author Contributions}

$\mathrm{XC}, \mathrm{WH}$ and $\mathrm{JH}$ designed the experiments; $\mathrm{XC}, \mathrm{WH}, \mathrm{LH}$ and JL performed the experiments; YF, XW, XX, ZW and KH provided help for the experiments; $\mathrm{XC}, \mathrm{WH}$ and $\mathrm{JH}$ analyzed the data; $\mathrm{XC}, \mathrm{WH}$ and $\mathrm{JH}$ wrote the manuscript.

\section{Competing Financial Interests}

The authors declare no competing financial interests.

\section{References}

1 Green DR, Levine B. To be or not to be? How selective autophagy and cell death govern cell fate. Cell 2014; 157:65-75.

2 Wallach D, Kang TB, Dillon CP, et al. Programmed necrosis in inflammation: toward identification of the effector molecules. Science 2016; 352:aaf2154.

3 Fuchs Y, Steller H. Programmed cell death in animal development and disease. Cell 2011; 147:742-758.

4 Wyllie AH. Glucocorticoid-induced thymocyte apoptosis is associated with endogenous endonuclease activation. Nature 1980; 284:555-556.

5 Shiokawa D, Maruta H, Tanuma S. Inhibitors of poly(ADP-ri- 
bose) polymerase suppress nuclear fragmentation and apoptotic-body formation during apoptosis in HL-60 cells. FEBS Lett 1997; 413:99-103.

6 Vanden Berghe T, Linkermann A, Jouan-Lanhouet S, et al. Regulated necrosis: the expanding network of non-apoptotic cell death pathways. Nat Rev Mol Cell Biol 2014; 15:135-147.

7 Labbe K, Saleh M. Cell death in the host response to infection. Cell Death Differ 2008; 15:1339-1349.

8 Zhang DW, Shao J, Lin J, et al. RIP3, an energy metabolism regulator that switches TNF-induced cell death from apoptosis to necrosis. Science 2009; 325:332-336.

9 He S, Wang L, Miao L, et al. Receptor interacting protein kinase-3 determines cellular necrotic response to TNF-alpha. Cell 2009; 137:1100-1111.

10 Cho YS, Challa S, Moquin D, et al. Phosphorylation-driven assembly of the RIP1-RIP3 complex regulates programmed necrosis and virus-induced inflammation. Cell 2009; 137:1112-1123.

11 Chen X, Li W, Ren J, et al. Translocation of mixed lineage kinase domain-like protein to plasma membrane leads to necrotic cell death. Cell Res 2014; 24:105-121.

12 Cai Z, Jitkaew S, Zhao J, et al. Plasma membrane translocation of trimerized MLKL protein is required for TNF-induced necroptosis. Nat Cell Biol 2014; 16:55-65.

13 Wang H, Sun L, Su L, et al. Mixed lineage kinase domain-like protein MLKL causes necrotic membrane disruption upon phosphorylation by RIP3. Mol Cell 2014; 54:133-146.

14 Dondelinger Y, Declercq W, Montessuit S, et al. MLKL compromises plasma membrane integrity by binding to phosphatidylinositol phosphates. Cell Rep 2014; 7:971-981.

15 Sun L, Wang H, Wang Z, et al. Mixed lineage kinase domain-like protein mediates necrosis signaling downstream of RIP3 kinase. Cell 2012; 148:213-227.

16 Zhao J, Jitkaew S, Cai Z, et al. Mixed lineage kinase domain-like is a key receptor interacting protein 3 downstream component of TNF-induced necrosis. Proc Natl Acad Sci USA 2012; 109:5322-5327.

17 Lamkanfi M, Dixit VM. Mechanisms and functions of inflammasomes. Cell 2014; 157:1013-1022.

18 Brennan MA, Cookson BT. Salmonella induces macrophage death by caspase-1-dependent necrosis. Mol Microbiol 2000; 38:31-40.

19 Cookson BT, Brennan MA. Pro-inflammatory programmed cell death. Trends Microbiol 2001; 9:113-114.

20 Shi J, Zhao Y, Wang K, et al. Cleavage of GSDMD by inflammatory caspases determines pyroptotic cell death. Nature 2015; 526:660-665.

21 Kayagaki N, Stowe IB, Lee BL, et al. Caspase-11 cleaves gasdermin $\mathrm{D}$ for non-canonical inflammasome signalling. $\mathrm{Na}$ ture 2015; 526:666-671.

22 He WT, Wan H, Hu L, et al. Gasdermin D is an executor of pyroptosis and required for interleukin-1 secretion. Cell Res 2015; 25:1285-1298.

23 Fink SL, Cookson BT. Caspase-1-dependent pore formation during pyroptosis leads to osmotic lysis of infected host macrophages. Cell Microbiol 2006; 8:1812-1825.

24 Ding J, Wang K, Liu W, et al. Pore-forming activity and structural autoinhibition of the gasdermin family. Nature 2016; 535:111-116.
25 Liu X, Zhang Z, Ruan J, et al. Inflammasome-activated gasdermin $\mathrm{D}$ causes pyroptosis by forming membrane pores. Nature 2016; 535:153-158.

26 Aglietti RA, Estevez A, Gupta A, et al. GsdmD p30 elicited by caspase-11 during pyroptosis forms pores in membranes. Proc Natl Acad Sci USA 2016; 113:7858-7863.

27 Quarato G, Guy CS, Grace CR, et al. Sequential engagement of distinct MLKL phosphatidylinositol-binding sites executes necroptosis. Mol Cell 2016; 61:589-601.

28 Daniele RP, Holian SK, Nowell PC. A potassium ionophore (Nigericin) inhibits stimulation of human lymphocytes by mitogens. J Exp Med 1978; 147:571-581.

29 Feinstein MB, Felsenfeld H. The detection of ionophorous antibiotic-cation complexes in water with fluorescent probes. Proc Natl Acad Sci USA 1971; 68:2037-2041.

30 Chen W, Zhou Z, Li L, et al. Diverse sequence determinants control human and mouse receptor interacting protein 3 (RIP3) and mixed lineage kinase domain-like (MLKL) interaction in necroptotic signaling. J Biol Chem 2013; 288:16247-16261.

$31 \mathrm{Wu}$ XN, Yang ZH, Wang XK, et al. Distinct roles of RIP1RIP3 hetero- and RIP3-RIP3 homo-interaction in mediating necroptosis. Cell Death Differ 2014; 21:1709-1720.

32 Roder P, Hille C. ANG-2 for quantitative $\mathrm{Na}(+)$ determination in living cells by time-resolved fluorescence microscopy. Photochem Photobiol Sci 2014; 13:1699-1710.

33 Bazzigaluppi P, Dufour S, Carlen PL. Wide field fluorescent imaging of extracellular spatiotemporal potassium dynamics in vivo. Neuroimage 2015; 104:110-116.

34 Reese AL, Kavalali ET. Spontaneous neurotransmission signals through store-driven $\mathrm{Ca}(2+)$ transients to maintain synaptic homeostasis. eLife 2015; 4:e09262.

35 Bashford CL, Alder G, Micklem KJ, et al. A novel method for measuring intracellular $\mathrm{pH}$ and potassium concentration. Biosci Rep 1983; 3:631-642.

36 Chan FK, Luz NF, Moriwaki K. Programmed necrosis in the cross talk of cell death and inflammation. Annu Rev Immunol 2015; 33:79-106.

37 Rodriguez DA, Weinlich R, Brown S, et al. Characterization of RIPK3-mediated phosphorylation of the activation loop of MLKL during necroptosis. Cell Death Differ 2016; 23:76-88.

38 Hildebrand JM, Tanzer MC, Lucet IS, et al. Activation of the pseudokinase MLKL unleashes the four-helix bundle domain to induce membrane localization and necroptotic cell death. Proc Natl Acad Sci USA 2014; 111:15072-15077.

39 Hou F, Sun L, Zheng H, et al. MAVS forms functional prion-like aggregates to activate and propagate antiviral innate immune response. Cell 2011; 146:448-461.

40 Xia B, Fang S, Chen X, et al. MLKL forms cation channels. Cell Res 2016; 26:517-528.

41 Pasantes-Morales H. Channels and volume changes in the life and death of the cell. Mol Pharmacol 2016; 90:358-370.

42 Maeno E, Ishizaki Y, Kanaseki T, et al. Normotonic cell shrinkage because of disordered volume regulation is an early prerequisite to apoptosis. Proc Natl Acad Sci USA 2000; 97:9487-9492.

43 Monteleone M, Stow JL, Schroder K. Mechanisms of unconventional secretion of IL-1 family cytokines. Cytokine 2015; 74:213-218. 
(Supplementary information is linked to the online version of the paper on the Cell Research website.) cc)(1)(8) $\Theta$ This work is licensed under a Creative Commons Attribution-NonCommercial-NoDerivs 4.0 Unported License. The images or other third party material in this article are included in the article's Creative Commons license, unless indicated otherwise in the credit line; if the material is not included under the Creative Commons license, users will need to obtain permission from the license holder to reproduce the material. To view a copy of this license, visit http://creativecommons.org/licenses/by-nc-nd/4.0/

(C) The Author(s) 2016 